\title{
Ohrverletzungen an Athleten auf Darstellungen des Altertums und ihre Beziehung zur medizinischen Literatur der Zeit*
}

\author{
Von J. Benedum, Gießen
}

«Keiner, der den Faustkampf wählt, erwartet, ohne Wunden einen Kranz zu erringen.» Solche Einsicht des Kirchenvaters Johannes Chrysostomus ${ }^{1}$ kennzeichnet schon die homerische Formulierung vom «schmerzensreichen Faustkampf $\gg{ }^{2}$ und Artemidor von DaLdis hält in seinem Traumbuch folgende Deutung für einen Faustkampftraum bereit:

«Das Faustkämpfen bringt jedermann Schlimmes; denn es bedeutet nebst Schmach auch Verluste; dabei wird nämlich sowohl das Gesicht verunstaltet als auch Blut vergossen, welches man als Geld betrachtet. Von Glück ist es nur für Leute, welche sich durch die Hantierung mit Blut ihren Lebensunterhalt verdienen, ich meine Ärzte, Opferpriester, Köche.» ${ }^{3}$

Den blutigen Verlauf von Boxkämpfen hat schon die Antike in Wort und Bild festgehalten. Die Skala der Verletzungen reicht dabei vom «blauen Auge» bis zum «Tod im Ring». ${ }^{4}$ Hier sollen jedoch nur die Ohrverletzungen interessieren, über deren Entstehung vielfach Unklarheit besteht. So schreibt L. ENGLERT ${ }^{5}$ : «Daß Verstümmlung der Ohren bei den griechi-

* Vortrag, gehalten in Schaffhausen am 30.September 1967 anläßlich der Jahrestagung der Schweizerischen Gesellschaft für Geschichte der Medizin und der Naturwissenschaften.

1 Ecloga de patientia, hom. 22, PG 63, 713: Nemo certare volens sine vulneribus coronam exspectat.

2 Ilias 23, 653. Vgl. Apollonius Rhodius, Argonautica 2, 76.

3 Oneirocritica 1, 61. Übersetzung nach F.S.Krauss, Artemidor von Daldis, Traumbuch, Basel 1965, S. 91.

4 Zum «blauen Auge» vgl. u.a. die Darstellung auf einer Schale mit Palästraszenen in Dresden bei C. BLÜMeL, Sport der Hellenen, Berlin 1936, Abb. 96, S. 127, und das Fragment einer Schale mit zwei Faustkämpfern in Berlin bei C. BlüMEL, a. a. O., Abb. 100, S. 31. Als literarisches Zeugnis darf besonders THEoKRIт, Idyll 22, 101 angeführt werden. Zum tödlichen Ausgang vgl. besonders den Boxkampf bei Apollonius RHodius, Argonautica 2, 1-98, und Valerius Flaccus, Argonautica 4, 148-314. Die grausigste Ausmalung eines «Todes im Ring» stellt ohne Zweifel der bei Pausanias 8, 40, 3 überlieferte Faustkampf dar. Dazu W.Rudolph, Olympischer Kampfsport in der Antike, Berlin 1965, S. $11 \mathrm{ff}$.

5 Untersuchungen zu Galens Schrift Thrasybulos, Studien zur Geschichte der Medizin, Heft 18, Leipzig 1929, S. 62. Vgl. auch F. Hauser, Gott, Heros und Pankratiast von Polyklet, Österreichische Jahreshefte 12 (1909). 110: "So unklar es auch unsereinem 
schen Athleten häufig vorkam, ist eine aus der Archäologie hinreichend bekannte Tatsache. Woher diese Verstümmlung kommt, darüber herrschen jedoch die merkwürdigsten Anschauungen.»

Ein Blick auf die griechischen und römischen Boxkampfdarstellungen ergibt, daß die Schläge fast ausnahmslos gegen Kopf und Gesicht erfolgten ${ }^{6}$, so daß in dieser Praxis geradezu eines der charakteristischen Kennzeichen antiken Faustkampfstiles erblickt werden darf $^{7}$. Da der Sieg nicht nach Punkten, sondern nur durch wirklichen oder technischen k.o. errungen werden konnte, mußte bestimmten Partien am Kopf besondere Bedeutung zukommen. So sollten Schläge gegen Mund, Nase und Augen die Kampfkraft des Gegners schwächen, Stöße gegen Kinn und Unterkieferwinkel den plötzlichen k. o.-Sieg herbeiführen. Daß dabei auch die Ohrpartie einer Gefährdung durch Schläge ausgesetzt sein mußte, dürfte aufgrund der schriftlichen und bildlichen Zeugnisse feststehen ${ }^{8}$.

Schon an den beiden ältesten literarischen Boxkampfdarstellungen bei HoMer fällt neben ihrer durchaus realistischen Wiedergabe die Technik des Niederschlages auf. In der Ilias wird der entscheidende Schlag gegen die Wange geführt, in der Odyssee gegen den Unterkieferwinkel und den

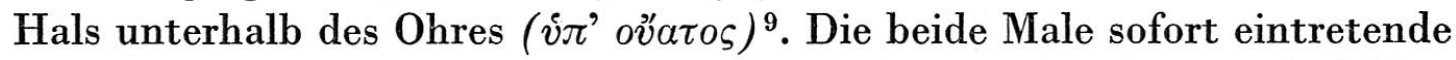
Ohnmacht kennzeichnet den Treffer, der noch heute beliebt ist, als Schlag

bleibt, auf welche Weise beim Ringen die bekannte Schwellung der Ohrmuschel entstehen könnte ...»

6 Vgl. J.H.Krause, Die Gymnastik und Agonistik der Hellenen, Leipzig 1841, I, 515.

7 Vgl. W.RudolPh, a.a.O., S. 11.

8 E.N.GARDINER, Greek athletic sports and festivals, London 1910, S. 425, vertritt die Ansicht, daß den Ohrverletzungen nur eine geringe Bedeutung beizumessen sei. Als Begründung weist er auf die literarische Tradition hin, in der verletzte Ohren kaum eine Rolle spielten. Die Vasenmalerei zeige darüber hinaus keine einzige Darstellung einer Ohrverletzung. Dem darf entgegengehalten werden, daß Ohrverletzungen in der schriftlichen Überlieferung keineswegs singulär sind. Vgl. die Stellen bei DE RIDDER, Artikel «Pugilatus», in Daremberg/Saglio, Dictionnaire des Antiquités, Graz 1963, IV, S.759, Anm. 26. Das Fehlen zerschlagener Ohren in der Vasenmalerei hat offenbar seinen Grund in den begrenzten Möglichkeiten dieses Genos. So findet man z. B. keine Boxernase oder auch nur irgendeine Form von Nasenverletzung, obwohl die Athleten einige Male aus der Nase bluten. Vgl. schon J.H. Krause, a.a. O., 517, Anm. 19.

9 Ilias 23, 690; Odyssee 18, 96. Die Homer-Nachahmung verrät der späte Nonvos AUS PANoplis, der seinen Boxkampf ebenfalls durch einen gezielten Fauststoß unter das Ohr

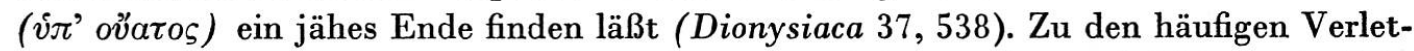
zungen im Halsbereich vgl. E. Prim, Hals, Nase und Ohr in Ilias und Odyssee, in Zeitschrift für Laryngologie, Rhinologie, Otologie und ihre Grenzgebiete 43 (1964) $330 \mathrm{ff}$. 
gegen die Carotis, die ihren Namen von dieser bei Faustkämpfen beobachteten Ohnmacht erhalten hat ${ }^{10}$. Schon hier muß gesagt werden, daß dieser Zielpunkt bei ungenauen Treffern Schläfe und Ohren in Mitleidenschaft ziehen mußte. In der Schilderung bei Apollonius Rhodius ${ }^{11}$ erfolgt der

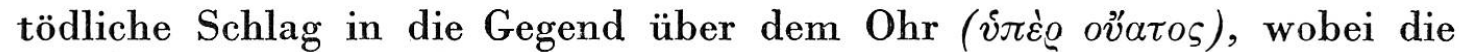
Splitter der Schläfenschuppe tief ins Gehirn eindringen. Schließlich wird bei der geradezu modern wirkenden Boxkampfreportage TheoкRits der eine der Gegner als ein Mann geschildert, «dessen Ohren von derben Fausthieben zerschlagen sind.$^{12}$ Die römischen Dichter Vergil, Martial und Valerius Flaccus ergänzen das Bild im nämlichen Sinn ${ }^{13}$.

Auf dem Hintergrund dieser literarischen Zeugnisse überrascht es nicht, wenn schon in der Antike Ohrverletzungen als Hauptmerkmal der Athleten

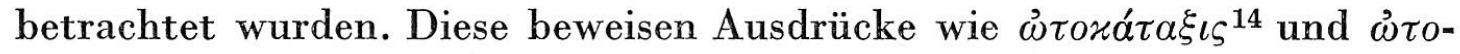
$\vartheta \lambda \alpha \delta \iota^{\prime} \alpha \varsigma^{15}$, deren sprachliche Grundlage das verunstaltete Ohr bildet, wenngleich sie den Athleten in seiner Gesamterscheinung meinen ${ }^{16}$. Die Entstehung solcher Traumen darf, wie wir gesehen haben, auf die häufigen Schläge gegen Halsschlagader und Schläfe zurückgeführt werden, wobei das äußere Ohr zumindest durch tangential auftreffende Schläge verletzt werden mußte ${ }^{17}$. An den Nachrichten aus dem Alterum fällt jedoch auf, daß nur von Traumen des äußeren Ohres die Rede ist, nie aber, soweit wir sehen, Verletzungen des inneren Gehörorgans Erwähnung finden. Selbst Schäden im Sinne einer commotio cerebri, die zum Erscheinungsbild der Encephalopathia traumatica führen ${ }^{18}$, werden nicht genannt. Bis zu welchem Grad freilich die Ohrmuschel in Mitleidenschaft gezogen werden konnte, vermag das Zeugnis eines Boxers trotz seiner damals wie heute charakteristischen Ruhmsucht zu verdeutlichen:

10 Vgl. u.a. L.A.KraUs, Kritisch-etymologisches medicinisches Lexikon, Göttingen 1844, 3. Auflage, S. 194, und E. MEHL, RE VII, A 2531, 25 ff.

11 Argonautica 2, 95.

12 THEOKRIT, Idyll 22, 45.

13 Vergil, Äneis 5, 435; Martial, epigr. 7, 32, 5; Valerius Flaccus, Argonautica 4, 309.

14 Vgl. Pollux, Onomasticon 2, 83.

15 Vgl. Diogenes Laertios 5, 67.

16 Vgl. Platon, Gorgias 515 e und Protagoras 342 b. Möglicherweise geht diesen Zeugnissen bereits Aristophanes frg. 98 voraus.

17 Vgl. J.H. Krause, a. a. O., S. 516: «Am härtesten aber wurden die Ohren mitgenommen »

18 Dazu F.HeIss, Unfallkunde und Therapie bei Sportverletzungen und Sportschäden, in Lehrbuch der Sportmedizin, herausgegeben von A. ARNOLD, Leipzig 1956, S. 584 f. 
«In Pisa verlor ich ein Ohr, in Platää ließ ich ein Auge, als (schein) tot trug man in Delphi mich fort. ${ }^{19}$

Derartige Entstellungen an Kopf und Gesicht mußten auch die bildende Kunst ${ }^{20}$, insbesondere die karikierende zur Darstellung reizen. So zeigt eine kleine hellenistische Arbeit aus Böotien ${ }^{21}$ (Abb. 1b) mit drastischer Deutlichkeit alle Merkmale, deren sich der Künstler beim Vorwurf einer Boxerkarikatur bedienen konnte. Neben der breiten Boxernase und den Glotzaugen fallen hier die unförmigen, überlappenden Klumpohren auf, die in der nebenstehenden Ringerkarikatur ${ }^{22}$ (Abb. 1a) wiederkehren. Man versteht beim Anblick dieser Figuren die Entrüstung des frommen Kirchenvaters Tertullian über solche Schändung des Ebenbildes Gottes, die er unter anderem in den «aurium fungi», den «Pilzohren» erblickte ${ }^{23}$. Der Ausdruck «Pilzohr» dürfte dabei auf eine traumatische Perichondritis hinweisen, an der die knorpeligen Teile des Cavum conchae und der Anthelix beteiligt sind. Ähnliches könnte hier vorliegen: Knórpelnekrose und narbige Schrumpfung scheinen zu einer Deformierung der Ohrmuschel geführt zu haben, wobei der Hautsack, seiner Knorpelstütze beraubt, nach Art eines Schlappohres nach vorne übergefallen ist. Mit einiger Sicherheit können wir also in den Krüppelohren dieser Boxerstatuette den er-

19 Lukillios, Anthologia Graeca, epigr. 11, 81, 2f. (ed. Beckвy). Vgl. auch Lukillios, a. a. O., epigr. 11, 78, 1f. (ed. BEсквy): «Dein Kopf ist ein Sieb ja geworden oder der Rand eines Buchs, den schon die Motten zernagt.»

'20 Die im folgenden herangezogenen Werke griechisch-römischer Kunst stellen eine bewußt paradigmatische Auswahl dar. Eine auch nur halbwegs vollständige Erfassung des in Frage kommenden archäologischen Materials erübrigte sich angesichts der medizinhistorischen Fragestellung. Dennoch seien hier einige weitere Bildnisse angeführt, die nach Ausweis ihrer «geschwollenen Ohren» als Athleten gedeutet werden, diese «Schwellung » aber nur teilweise mit klinischen Erscheinungsbildern in Einklang bringen lassen: Jüngling von Tralles (vgl. H. Sichtermann, Antike Plastik, Berlin 1965, Lieferung IV, Teil 9, Tafel 47). Athletenproträt im Konservatoren-Palast (ebenda Abb. 10 und 11). Bärtiger Kopf in Rom (vgl. K. Schauenburg, Antike Plastik, Berlin 1963, Lieferung II, Teil 5, Tafel 46-48). Herakles in Magdeburg (ebenda Tafel 49-53). Athletenkopf aus Lucus Feroniae (vgl. T.Dohrn, Antike Plastik, Berlin 1967, Lieferung VI, Teil 6, Tafel 42-47). Athletenkopf in Berlin (vgl. C. BLÜMeL, Sport und Spiel bei Griechen und Römern, Berlin 1934, S. 49, Abb. 33) unter vielen anderen.

21 C. Blümel, Sport der Hellenen, Berlin 1936, Abb. 53, Beschr. S. 19.

22 C. BlüMel, a. a. O., Abb.52, Beschr. S. 19.

${ }^{23}$ Tertullian, de spectaculis 23: «Tales enim cicatrices caestuum et callos pugnorum et aurium fungos a Deo cum plasmate accepit?» 
wähnten «aurium fungus » ${ }^{24}$, das heutige «Blumenkohlohr», wiedererkennen ${ }^{25}$.

Erleichterte uns die übersteigerte Bildaussage der Karikatur eine Diagnose, so treten in den Darstellungen der Plastik sofort Identifikationsschwierigkeiten auf. Ein sicheres Kriterium für eine Ohrverletzung läßt sich bei dem um 560/550 v. Chr. entstandenen Boxerrelief ${ }^{26}$ (Abb.2) im Kerameikos-Museum in Athen nicht erbringen. Zwar könnte die «Adlernase» des kurzgeschorenen, bärtigen Mannes, der nach Ausweis seiner umgürteten Linken ohne Zweifel ein Boxer war, als Zeichen seines Berufes gedeutet werden, das strukturell nur unvollkommen gearbeitete $\mathrm{Ohr}$ aber dürfte auf den weich-quellenden Stil dieser Zeit zurückzuführen sein, ohne daß freilich eine echte Verletzung etwa im Sinne eines Othämatom völlig ausgeschlossen werden kann. Auffällig mag immerhin das Verstreichen von Helix und Anthelix, von Tragus und Antitragus bleiben.

Ähnlich steht es mit dem berühmten Kopf Rayet ${ }^{27}$ (Abb. 3) in Kopenhagen, einer attischen Arbeit aus derselben Periode um 540/530 v. Chr. Hier wird der Eindruck einer Ohrschwellung neben den teigigen Formen dieses Stils zusätzlich durch die äußere Beschädigung verstärkt. So schreibt C. BLÜMEL ${ }^{28}$ : «Nach den verschwollenen Ohren zu urteilen, gehörte der Kopf wahrscheinlich zur Grabstatue eines Faustkämpfers.» Wie zuvor bedeutete es aber auch hier eine Verkennung des Stils in naturalistischem Sinn, wollte man von einer eindeutigen Deformierung des Ohres sprechen ${ }^{29}$.

24 fungus (= Pilz, Schwamm) gebraucht z. B. Celsus 6, 18, 11 (p. 297, Marx) für ein Geschwür am Mastdarm bzw. Uterus. Im Griechischen trägt der Plural ( $\sigma \pi o ́ \gamma \gamma o \iota)$ die Bedeutung von Tonsille. Vgl. HippoKrates, epid. 4, 7 (V 148 L.).

25 Darüber hinaus zeigt der massige Körper der Boxerstatuette die bekannte Fettleibigkeit der Schwerathleten, die durch systematische Zwangsdiät (ảvayxopayía) erreicht wurde. Nach Philostrat (de gymnastica 34) soll eine Dickbäuchigkeit sogar die Härte der Schläge gegen das Gesicht mildern können. Die von Sokrates (Xenophon, Symposium 2, 14) getadelten dicken Schultern und dünnen Beinchen der Faustkämpfer treffen wohl nur für die Karikatur zu, besonders augenscheinlich an einem Ringerpaar auf einem Becher aus dem Kabirion bei Theben (B. SchröDER, Der Sport im Altertum, Berlin 1927, Abb.12, und C.Blümel, Sport der Hellenen, Berlin 1936, Abb.130, Beschr. S. 37). Auch hier erkennen wir keine Deformierung der Ohren, wie überhaupt in der ganzen Vasenmalerei weder ein Athletenohr noch eine Boxernase nachweisbar sind.

26 G.M.A. Richter, The archaic gravestones of Attica, London 1961, Abb. 92, Beschr. S. $23 \mathrm{f}$.

27 R.Lullies/M. Hirmer, Griechische Plastik, München 1960², Tafel 46, Beschr. S. 46.

28 Sport der Hellenen, Berlin 1936, S. 21.

29 Vgl. V.H.Poulsen, Three archaic heads in the Ny Carlsberg Glyptotek, in From the Collections of the Ny Carlsberg Glyptotek, 1938 II, Kopenhagen 1939, besonders S. 96 ff., 
Auch für die inschriftlich als Themistokles gesicherte Herme aus Ostia ${ }^{30}$, die römische Kopie eines griechischen Originals, das um 460 entstanden sein mag, ist eine Ohrverletzung behauptet worden ${ }^{31}$. Eine Detailaufnahme ${ }^{32}$ (Abb. 4) zeigt jedoch, daß keine traumatische Veränderung der Ohrmuschel vorliegt. Die eigenwillige Form des Ohres findet vielmehr Parallelen in der Plastik der gleichen Zeit ${ }^{33}$.

Hierher gehört auch der Bronzekopf eines Siegers aus Olympia ${ }^{34}$ (Abb.5) aus der Mitte des 4. Jahrhunderts v. Chr. Neben der ornamentalen Anordnung von Haar und Bart fallen besonders die tiefliegenden Brauen und die breite Nase auf, die für einen Athleten sprechen können. Eine Überprüfung der in der Archäologie regelmäßig als Beweis für einen Faustkämpfer herangezogenen Ohren ${ }^{35}$ wird aber dadurch erschwert, daß keine der beiden Seitenansichten ein freiliegendes Ohr zeigt. Dennoch wird der Anschein einer starken Verstümmlung erweckt. Endgültige Klarheit kann aber hier nur eine Betrachtung am Original schaffen.

Das prägnanteste Beispiel einer antiken Boxerstatue stellt der sitzende Faustkämpfer im Thermenmuseum in Rom ${ }^{36}$ dar. Der tadellose Erhaltungszustand dieses Bronzewerkes gibt nicht nur Aufschluß über Form und Aussehen der gefährlichen ledernen Schlagringe, sondern zeigt zugleich die kraß-realistische Wiedergabe eines Boxergesichts ${ }^{37}$ (Abb. 6): breitgeschlagener Nasenrücken, wulstig aufgetriebene Augenbrauen, Platz-

und R.Lullies/M.Hirmer, a.a.O., Beschr. S. 46. Ferner G.M.A. Richter, Kouroi, London 1960, Beschr. S. 120.

${ }^{30}$ G.M.A. Richter, Greek portraits, A study of their development, Latomus 20 (1955) Tafel I, Abb. 1 und 2. Dieselbe, The portraits of the Greeks, London 1965, Abb. 405-408.

${ }^{31}$ Literaturnachweis bei H.Sichtermann, Der Knabe von Tralles, Antike Plastik, Berlin 1965, Lieferung IV, Teil 9, S. 72, Anm. 7.

${ }^{32}$ G.M.A. Richter, Greek portraits, Tafel II, Abb. 4.

${ }^{33}$ G.M.A. Richter, Greek portraits, S. 17 f. Vgl. Tafel II, Abb. 3 und 5.

${ }^{34}$ E.Schmidt, Silanion der Meister des Platonbildes, Jb. dtsch. Arch. Inst. 49 (1934) 194, Abb. 5.

${ }^{35}$ Vgl. u. a. R. KéKulé v. Stradonitz, Über den Bronzekopf eines Siegers in Olympia, Sitzber. Preuß. Akad. Wiss. phil.-hist. Kl. 26 (1909) 699. C.BlüMel, Sport der Hellenen, Berlin 1936, Beschr. S. 12, und R.Luldies/M.Hirmer, Griechische Plastik, München $1960^{2}$, Beschr. S. 82. Ferner E. HolläNDER, Plastik und Medizin, Stuttgart 1912, S. 309 f.

36 W. Helbig/W. Amelung, Führer durch die öffentlichen Sammlungen Klassischer Altertümer in Rom, Leipzig $1913^{3}$, 2. Band, Nr. 1350, 136 ff. Vgl. auch C. BlüMel, Sport der Hellenen, Berlin 1936, Abb. 12, Beschr. S. 11.

37 W.H.Schuchhard, Die Kunst der Griechen, in: Geschichte der Kunst, Altertum, Berlin 1940, S. 447, Abb. 416. 


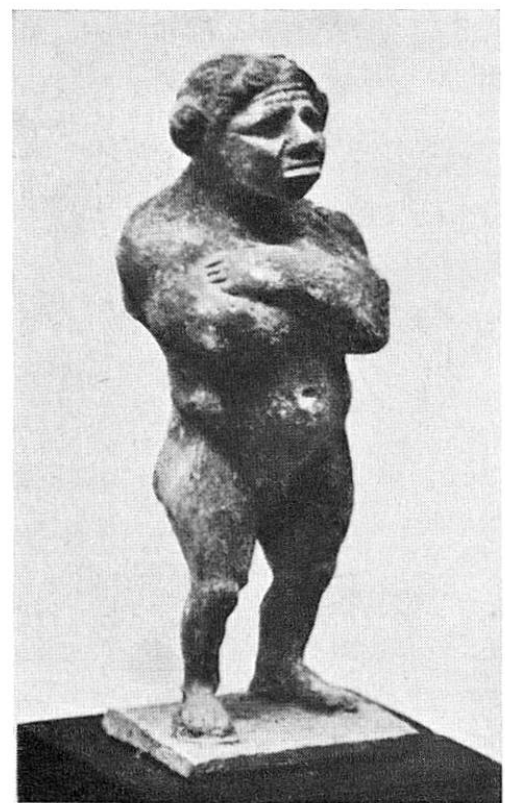

Abb. 1 a

Statuette eines Ringers (?)

Museum Karlsruhe

Hellenistische Arbeit

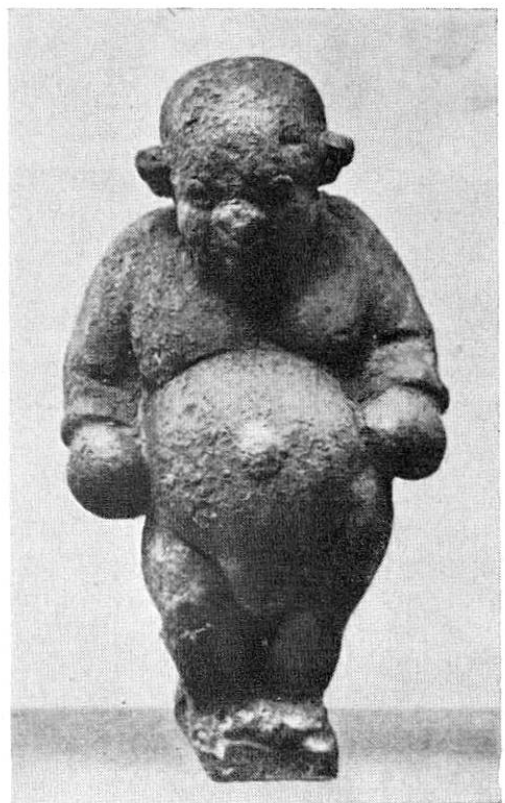

Abb. 1b

Statuette eines Faustkämpfers Museum Dresden

Hellenistische Arbeit

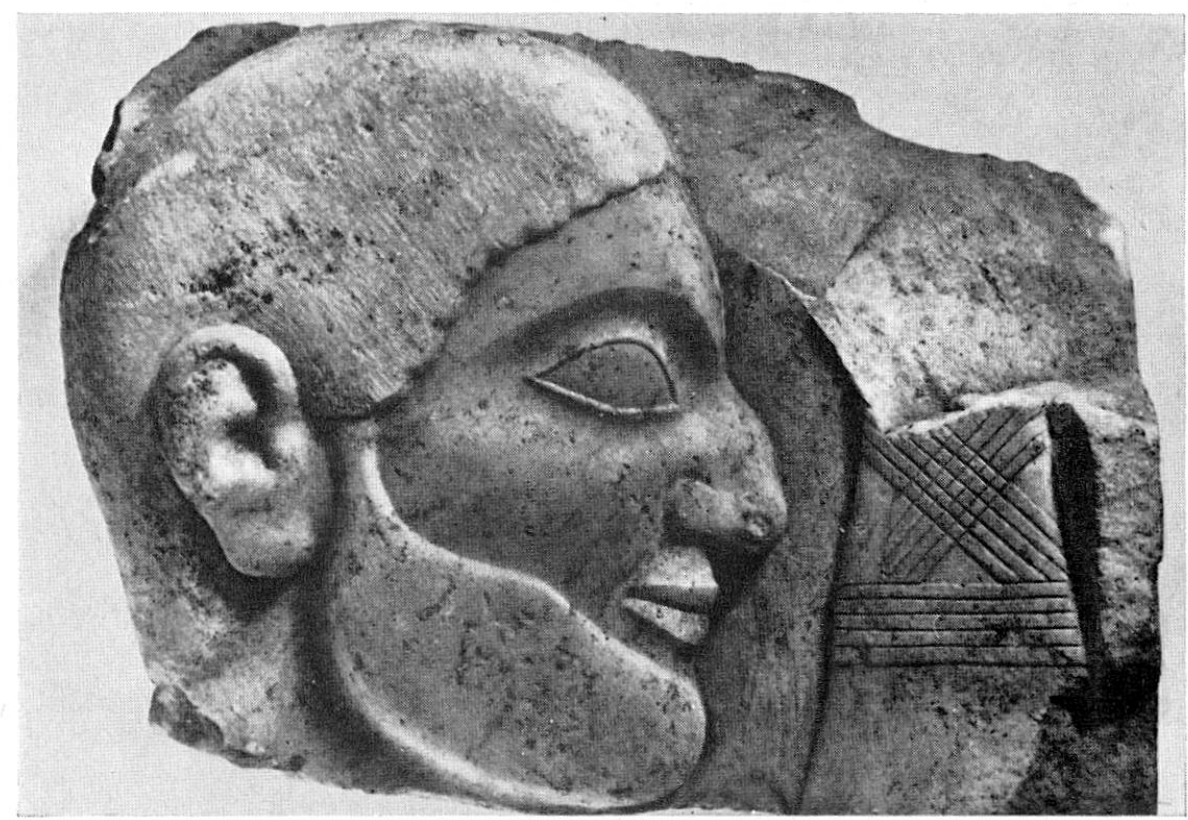

Abb. 2

Relief eines Faustkämpfers

Kerameikos-Museum, Athen, 560/550 v. Chr. 


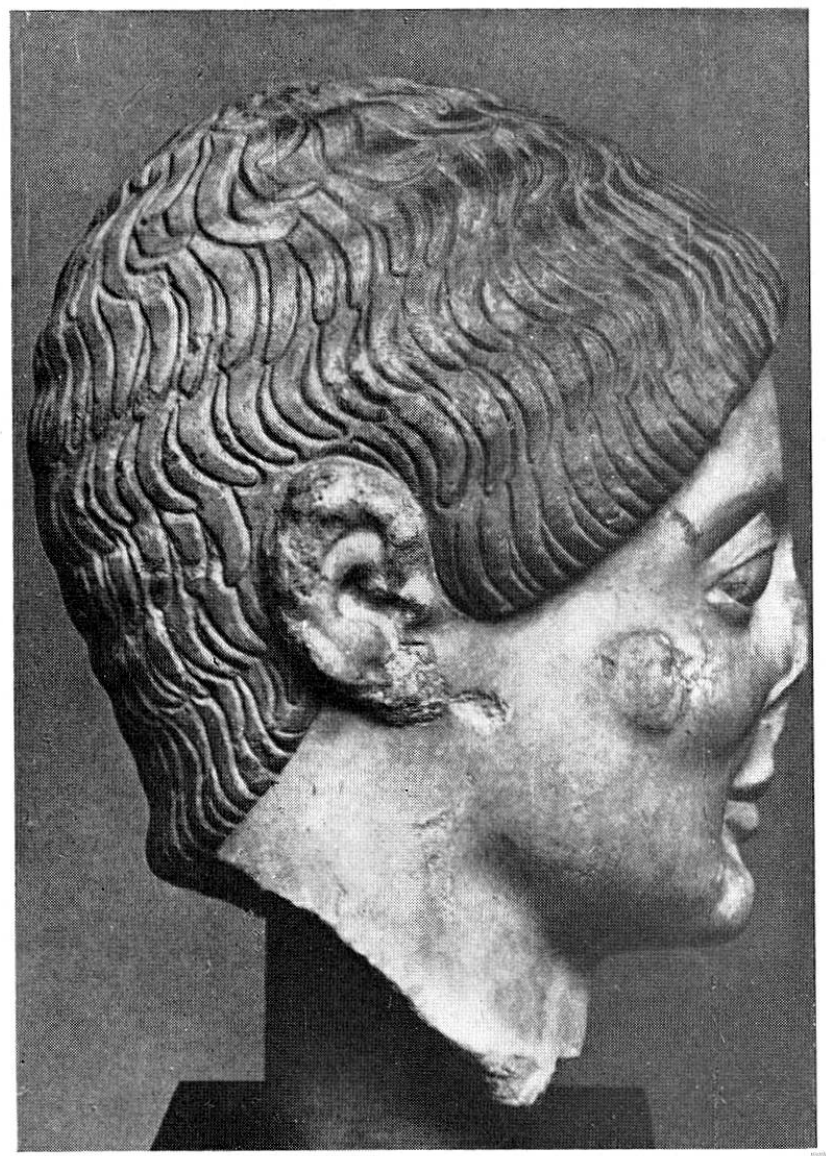

Abb. 3

Kopf Rayet

Ny-Carlsberg-Glyptothek,

Kopenhagen

$540 / 530$ v. Chr.

Abb. 4

Themistoklesherme

Museum Ostia

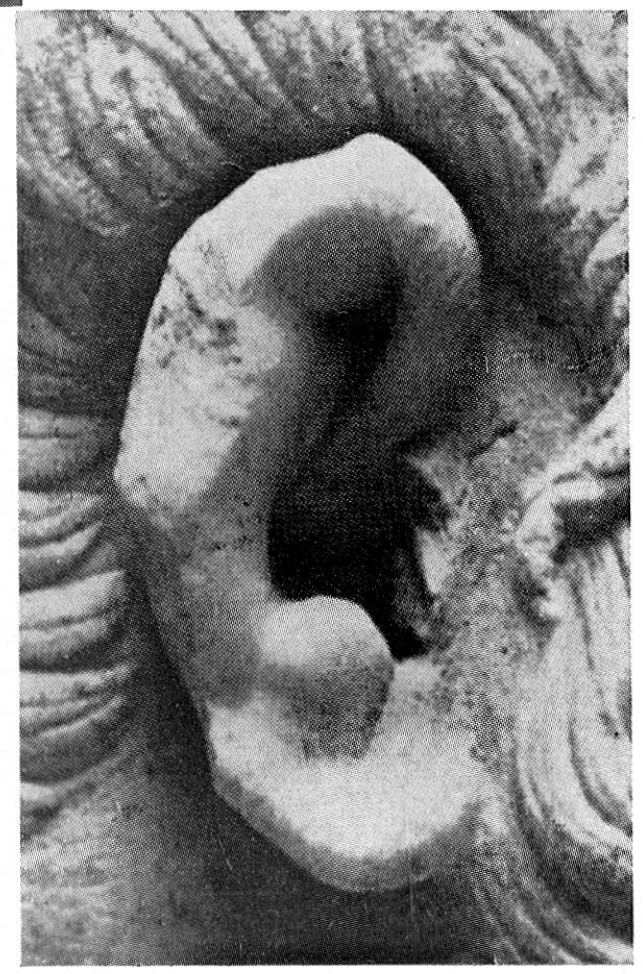

um 460 v. Chr. 
Abb. 5

Bronzekopf eines Faustkämpfers National-Museum, Athen Mitte des 4. Jahrhunderts v. Chr.

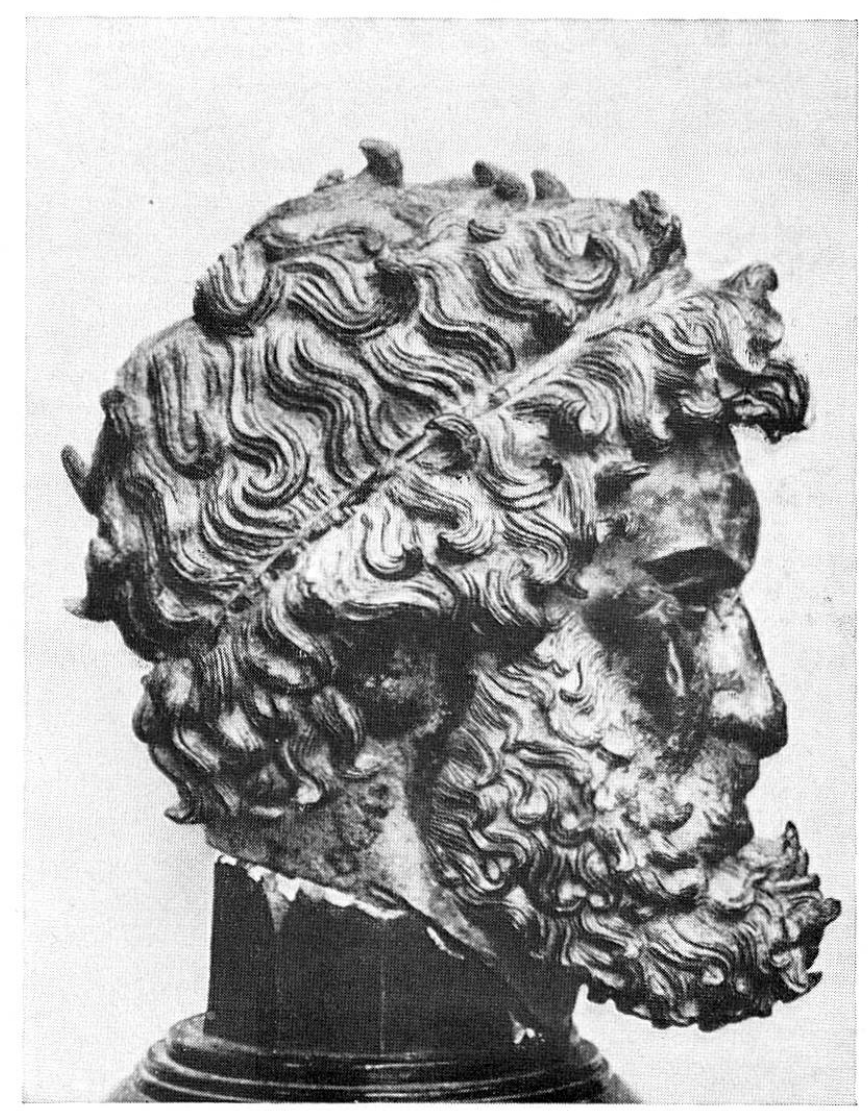

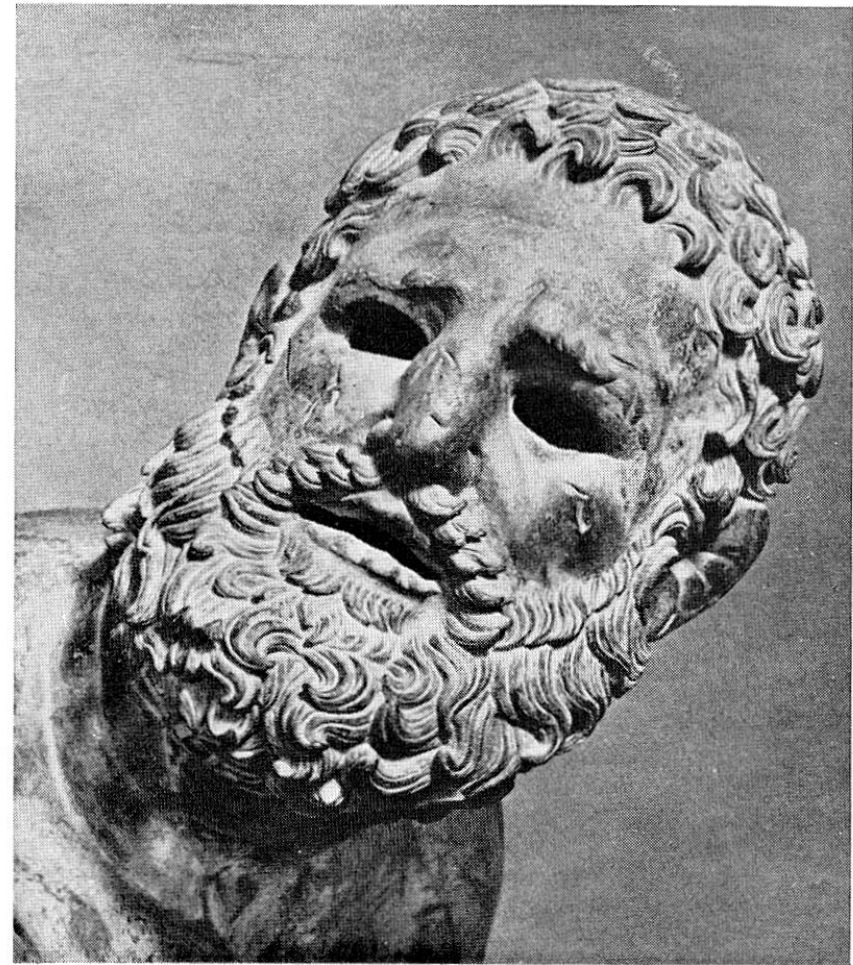

Abb. 6

Faustkämpfer

Museo Nazionale Romano, Rom Mitte des 1. Jahrhundertsv. Chr. 


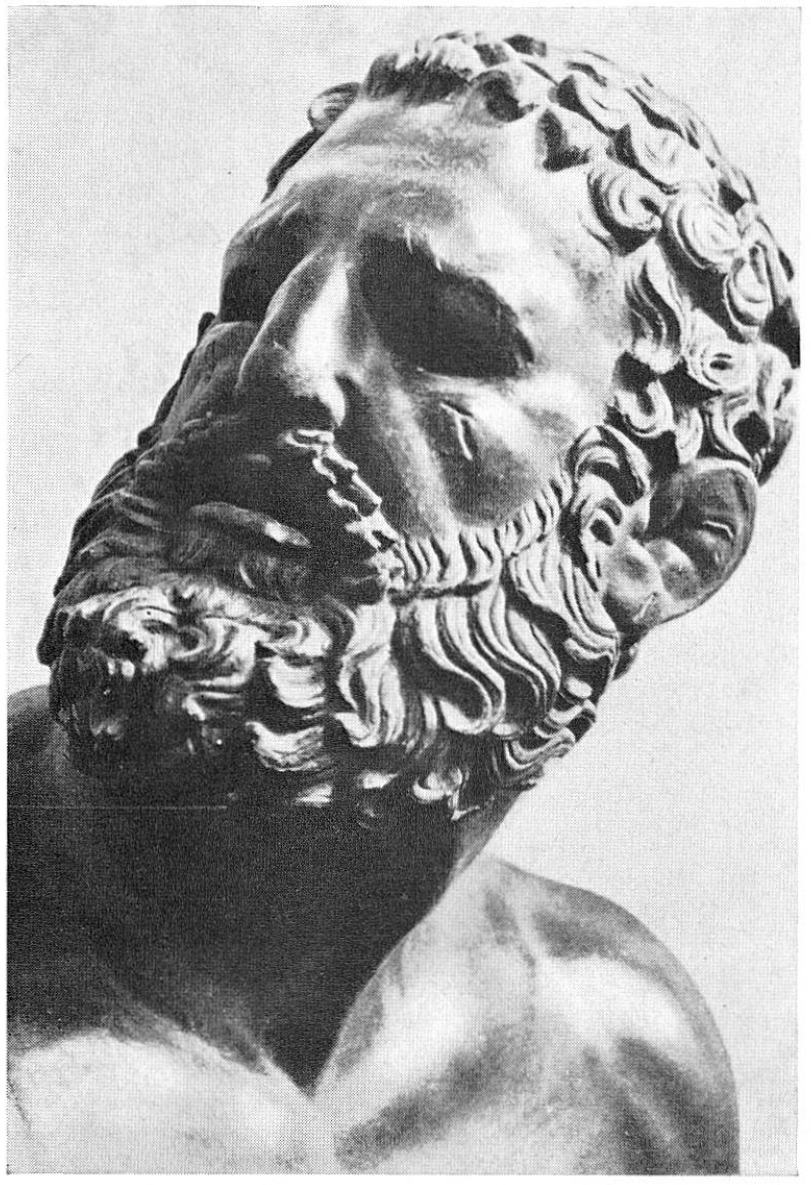

Abb. 7

Faustkämpfer des Apollonios Museo Nazionale Romano, Rom Mitte des 1. Jahrhundertsv. Chr.

Abb. 8

Römischer Athlet

Museum Berlin

Ende des 3. Jahrhunderts n. Chr.

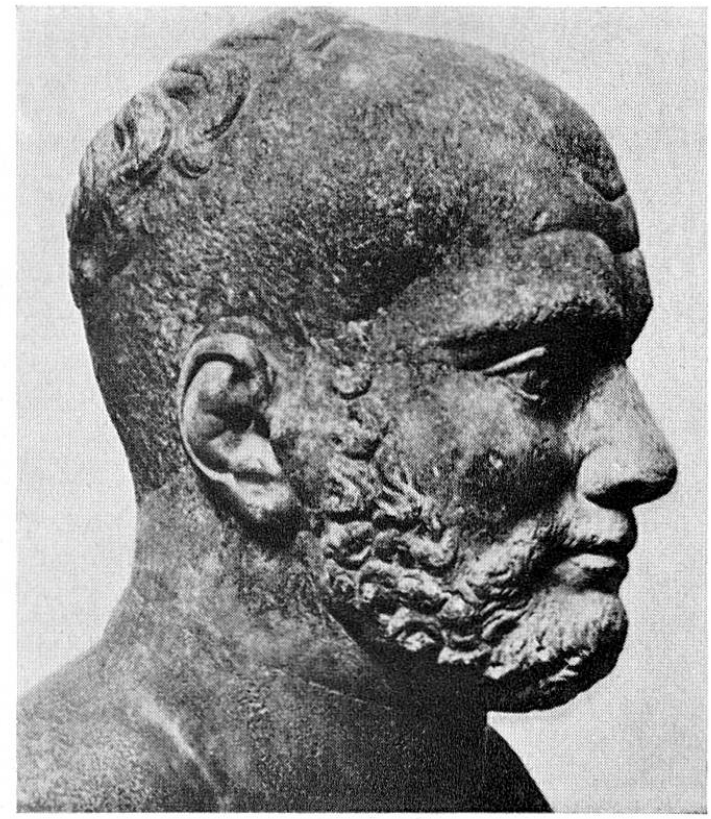


wunden an Wange, Nase und Ohr. Die leicht zurücktretende Oberlippe deutet darauf hin, daß die obere Zahnreihe eingeschlagen ist. Ebenso läßt der halb geöffnete Mund vermuten, daß eine Atmung durch die Nase erschwert ist. Der tierisch-brutale Gesichtsausdruck spricht für sich selbst. Besondere Beachtung verdient das verunstaltete $\mathrm{Ohr}^{38}$ (Abb. 7). Mehrfache «décollements traumatiques» müssen hier vorangegangen sein, die Haut und Perichondrium vom Knorpel gelöst und zur Bildung eines Othämatoms geführt haben. Als Folge dürfte die Ohrmuschel höckrig geworden sein, jedenfalls scheint das entstandene Narbengewebe die Haut straff am Knorpel fixiert zu haben. Der radiären Faltung der Ohrmuschel könnte dabei eine mehrfache Knorpelfraktur zugrundeliegen. Zusätzlich zu diesen Traumen sind frische Schlagverletzungen vorhanden; denn der Künstler hat Wunden dargestellt, aus denen frische Blutstropfen quellen ${ }^{39}$. Mit einiger Wahrscheinlichkeit dürfen wir also an diesem Faustkämpferohr die bewußte Darstellung eines alten Othämatoms ${ }^{40}$ erkennen, dem zusätzlich frische Verletzungen beigebracht sind.

Einen Athleten mit ausgeprägter Ohrverletzung kann schließlich ein Porträt aus der späten Kaiserzeit, dem Ende des 3.Jahrhunderts n.Chr., zeigen (Abb. 8). Der Kopf ${ }^{41}$, dessen Nase ergänzt ist, weist eine deutliche

38 C.BlüMEL, Sport der Hellenen, Berlin 1936, Abb. 12, Beschr. S. 11.

39 R.Lullies und M.Hirmer, Griechische Plastik, München $1960^{2}$, Beschr. S. 93: «Die Ohren sind von Hieben verschwollen und mit frischen Wunden bedeckt, aus denen Blutstropfen hervorquellen. » Vgl. L. ALscher, Griechische Plastik IV, Berlin 1957, S. 136 : «Damit sowie mit den geschwollenen Ohren und der geplatzten Haut der Wangen ist der Kopf von Spuren des grausamen Kampfes gezeichnet. »

$40 \mathrm{Vgl}$. H.G.Boenninghaus, Ohrverletzungen, in Hals-, Nasen- und Ohrenheilkunde, herausgegeben von J.Berendes, R.Link und F.Zöllner, Band III/1, Stuttgart 1965, S. 836.

Eine solche Verbildung der Ohren auf der Grundlage von Othämatomen, denen in der Regel ein stumpfes Trauma durch tangentiale Krafteinwirkung vorangeht, ist noch heute als Berufskrankheit bei Metzgern, Zimmerleuten, Lastträgern, Ringern und Boxern bekannt. Virchow sah Ähnliches bei japanischen Ringern, Valentin bei schweizerischen «Schwingern» und Bloch bei «Saltomortalefängern» (vgl. W.KümmEL, Die Chirurgie des Ohres, in Handbuch der praktischen Chirurgie, herausgegeben von P. von Bruns, C. Garré und H.Küttner, Band 1: Chirurgie des Kopfes, Stuttgart 1913, S. 386). Die häufige Beobachtung von Othämatomen an Insassen von Irrenanstalten erklärt sich durch Mißhandlungen seitens der Wärter. Meist ist davon das Ohr der linken Seite betroffen, weil diese die «Ohrfeigenseite» darstellt. Vgl. Real-Encyclopädie der gesammten Heilkunde, herausgegeben von A. Eulenburg, Wien 1898, Band 17, S. 497.

41 C.Blümel, Sport und Spiel bei Griechen und Römern, Berlin 1934, Abb. 47. 
Atrophie des Ohres auf. Die Konturen der Anthelix sind verstrichen und von radiären Falten durchbrochen. Auch hier dürfte ein ausgedehntes Othämatom vorliegen.

In der medizinischen Literatur hören wir erstmals in der hippokratischen Schrift De articulis $^{42}$ von Verletzungen der Ohrmuschel, für die zwar die Palaestra als Entstehungsort nicht angegeben ist, aber wie bei zahlreichen anderen Verletzungen in dieser Schrift fraglos angenommen werden darf. Der Verfasser bespricht hier ausführlich die Ohrknorpelfraktur ${ }^{43}$ und verwirft, ähnlich wie beim Nasenbeinbruch, die herkömmlichen Bandagen. Er empfiehlt entweder überhaupt keinen Verband ${ }^{44}$ oder höchstens eine Auflage von klebendem Mehlteig ${ }^{45}$. Ist es zu einer eitrigen Entzündung mit Einschmelzung gekommen, so muß an dem erhabenen Punkt der Fluktuation eine Inzision vorgenommen werden, um dem Eiter Abfluß zu verschaffen. Am raschesten tritt eine Heilung ein, wenn der Knorpel durchbrannt wird. «Doch wisse man wohl, daß, wenn durch und durch gebrannt wird, das $\mathrm{Ohr}$ verkrüppelt und kleiner sein wird als das andere. $\rangle^{46}$

Einen wesentlichen Fortschritt in der Behandlung von Ohrknorpelbrüchen bringt Celsus ${ }^{47}$, der damit ohne Zweifel den Stand der alexandrinischen Chirurgie widerspiegelt. Solange keine Eiterung auftritt, legt er bei Knorpelbrüchen ohne Hautverletzung ein Medikament auf, das die Wiedervereinigung der getrennten Knorpelreste fördern soll. Besonderen Wert legt er dabei auf die Tatsache, daß der Knorpel keinen Callus bildet, die Fragmente vielmehr durch Granulationen wieder verbunden werden.

42 De art. c. 40 (II, 161 ff., KÜHLEWEIN).

43 Vgl. Ps. Galen, introd. c. 20 (XIV, 792 K.): «Auch Ohrbrüche begegnen nach Hippokrates und weil sich der Knorpel ähnlich wie der Knochen verhält, kann er nur sehr schwer in die ursprüngliche Form reponiert werden.»

44 Vgl. Paulus v. Aegina III 23, 12 (cmg IX, 1, p. 192).

${ }^{45}$ Im Mochlikon c. 3 (II, 248, K.) wird statt dessen Cerat oder klebende Schwefelsalbe empfohlen. Vgl. K. DeichgräBer, Die Epidemien und das Corpus Hippocraticum, $A b h$. Preuß. Akad. Wiss. phil.-hist. Kl., Nr. 3, Berlin 1933, S. 87.

46 De art. c. 40 (II, 162, K.). Vgl. Mochlikon c. 3 (II, 248, K.). Es überrascht dabei nicht, daß das operative Verfahren durch einfache Inzision bzw. Durchbrennung des Knorpels zur narbigen Schrumpfung führt. Erst die moderne Chirurgie kennt Methoden zur Verhütung von Entstellungen nach Ohrmuschelperichondritiden. Vgl. u.a. A.ToBEcK, Beitrag zur operativen Behandlung der Perichondritis der Ohrmuschel, in Z. Hals-, Nasenu. Ohrenheilkde. 44 (1938) 368-373. Vgl. auch H.G.Boenninghaus, Ohrverletzungen, in Hals-, Nasen- und Ohrenheilkunde, herausgegeben von J.Berendes, R. Link und F.ZöLLNER, Band III/1, Stuttgart 1965, S. 838.

47 Celsus 8, 6 (p. 383 f., Marx). 
Tritt eine eitrige Entzündung hinzu, so muß der betroffene Knorpel mit einem halbmondförmigen Schnitt herausgetrennt werden.

Bei diesem schon differenzierteren Verfahren vermißt man einen Hinweis auf die Gefahr der Deformierung, die eine Knorpelresektion zwangsläufig zur Folge hat ${ }^{48}$. Auch hier fehlt eine Angabe über Ursache und Vorkommen der Ohrknorpelbrüche, doch auch in dieser Zeit werden die Athleten den Hautpanteil solcher Verletzungen gestellt haben ${ }^{49}$.

Einen weit tieferen Einblick in die Operationsmethoden bei Ohrerkrankungen, die innerhalb der alexandrinischen Chirurgie ihre Entwicklung gefunden haben müssen, gestattet das 9. Kapitel des 7. Buches, «die älteste im Abendland verfaßte Abhandlung über plastische Chirurgie, die chirurgia curtorum $»{ }^{50}$ Celsus gibt hier ein Verfahren an, mit dem kleine Kolobome, die angeboren oder erworben sind, an Ohren, Lippen und Nase wieder ausgeglichen werden können. Die Methode umfaßt daher auch die Ohrverletzungen mit Hautdefekt (Abb. 9): Der Substanzverlust wird zu einem Viereck vervollständigt, und der obere und untere Schnitt nach beiden Seiten verlängert. Die so gelösten Hautteile werden zur Mitte des Defektes herangezogen und durch Naht zur Vereinigung gebracht. Sollten durch die zusammengezogenen Hautlappen Deformierungen in der Nachbarschaft eintreten, werden halbmondförmige Entspannungsschnitte ${ }^{51}$ gelegt. Damit ist jene als «méthode française par déplacement ${ }^{52}$ bekannte Transplantation durch seitliche Verschiebung bereits als antik erwiesen ${ }^{53}$.

Die wenigen inhaltsarmen Zeugnisse über die Ohrverletzungen aus der pneumatischen Chirurgie lassen gegenüber Celsus keinen wesentlichen Fort-

48 Möglicherweise soll die Fixierung der Ohrmuschel durch weiche Wolle von hinten zwischen Ohr und Schädel und einen lockeren Verband von vorn einer groben Verunstaltung wie beispielsweise einer Knickung entgegenwirken.

${ }^{49} \mathrm{Vgl}$. Celsus, Über die Arzneiwissenschaft, übersetzt und erklärt von E. Scheller, Braunschweig $1906^{2}$, S. 768, und J.Ilberg, A.Cornelius Celsus und die Medizin in Rom, Jahrbücher für das Klassische Altertum 19 (1907) 401.

50 E. Gurlt, Geschichte der Chirurgie, Hildesheim 1964, I, S. 359.

${ }^{51}$ Inwieweit die halbmondförmigen Entspannungsschnitte Dieffenbachs hiermit in Zusammenhang stehen, bedarf einer eigenen Untersuchung.

${ }^{52}$ Vgl. M.SerRe, Traité sur l'art de restaurer les difformités de la face selon la méthode par déplacement ou méthode française, Montpellier 1842, und PH. Rigaud, De l'anaplastie des lèvres, des joues et des paupières, Paris 1841. Zur sogenannten «indischen» und «italienischen Methode» vgl. Real-Encyclopädie der gesammten Heilkunde, herausgegeben von A. Eulenburg, Wien $1894^{3}$, Band 2, S. 564.

${ }^{53}$ Vgl. schon J.Hirschberg, Geschichte der Augenheilkunde im Altertum, in GraefeSaEmisch, Handbuch der gesamten Augenheilkunde, Band XII, Leipzig 1899, S. 290. 


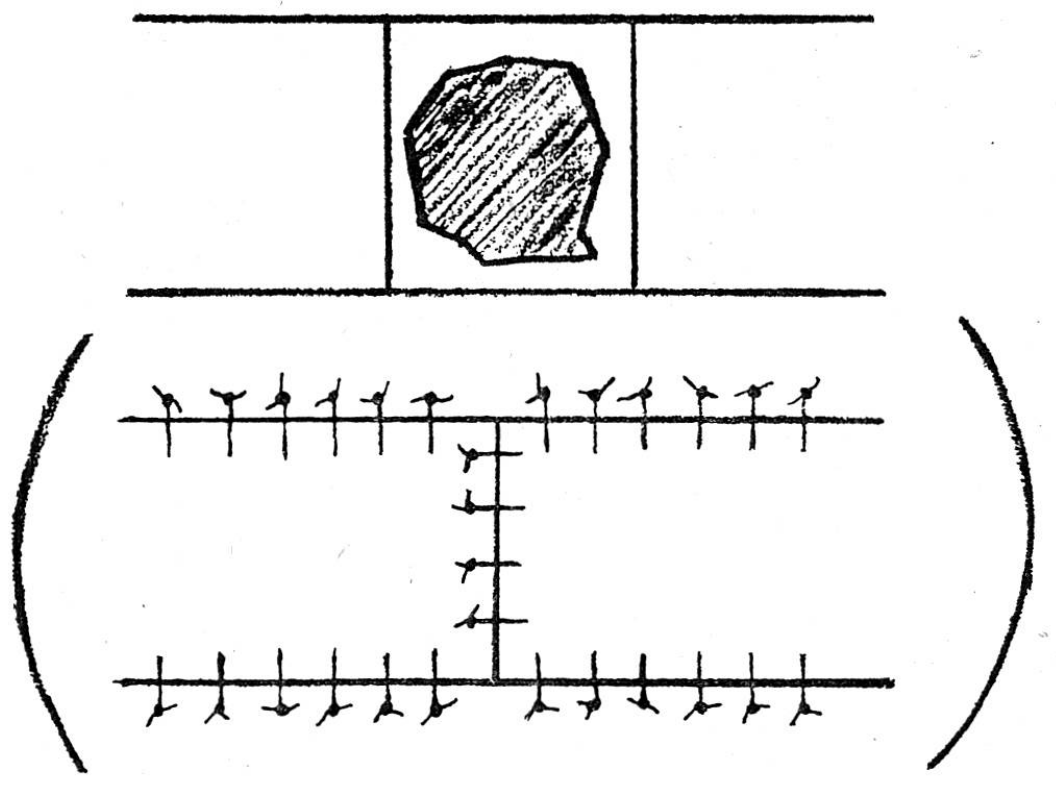

Abb. 9. Transplantation durch seitliche Verschiebung mit halbmondförmigen Entlastungsschnitten nach Celsus

schritt erkennen. In der vermutlich frühpneumatischen ${ }^{54}$ pseudogalenischen Introductio gibt der Verfasser eine kurze Anweisung zur Wiederherstellung von Kolobomen an Nase, Ohren und Lippen: «Bei Defekten an Ohren und Nase muß man den Knorpel, wenn er gebrochen ist, excidieren $^{55}$ und die Haut mit einem wollenen Faden zusammennähen » ${ }^{56}$. Ebenso rät Archigenes aus Apameia bei Wunden und Brüchen des Ohres zu einer Reposition $^{57}$ des Knorpels mit anschließender Knopfnaht ${ }^{58}$. Einen Fort-

54 Vgl. J.Kollesch, Zur Geschichte des medizinischen Lehrbuchs in der Antike, in Verhandlungen des XIX. Internationalen Kongresses für Geschichte der Medizin, Basel 1966, S. 203-208.

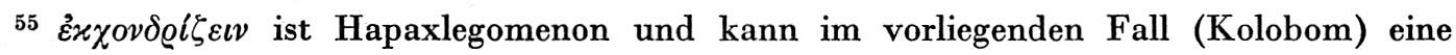
geringfügige Knorpelresektion meinen, die eine allzu große Spannung der Hautnaht verhindern soll.

56 Ps. Galen, introd. c. 19 (XIV, 791, K.).

${ }^{57} \varepsilon \dot{\gamma} \chi o v \delta \varrho i \zeta \varepsilon \iota v$ ist Hepaxlegomenon und scheint die Adaptierung der getrennten Knorpelenden zu meinen.

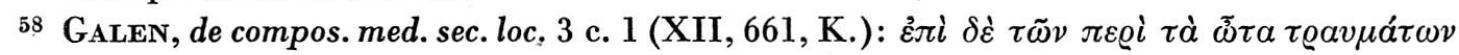

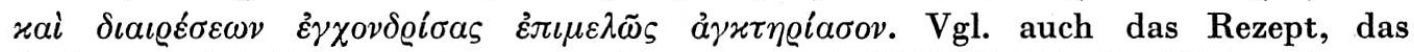

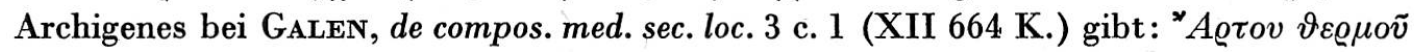

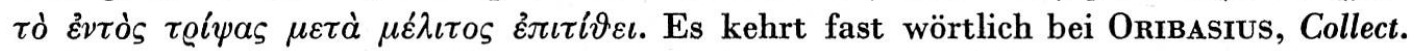
med. ecl. med. 10,4 (cmg VI, 2, 2, p. 189), Alexander von Tralles, 3 c. 2 (II, p.93, Puschmann) und Paulus von Aegina III 23, 12 (cmg IX, 1, 192) wieder. 

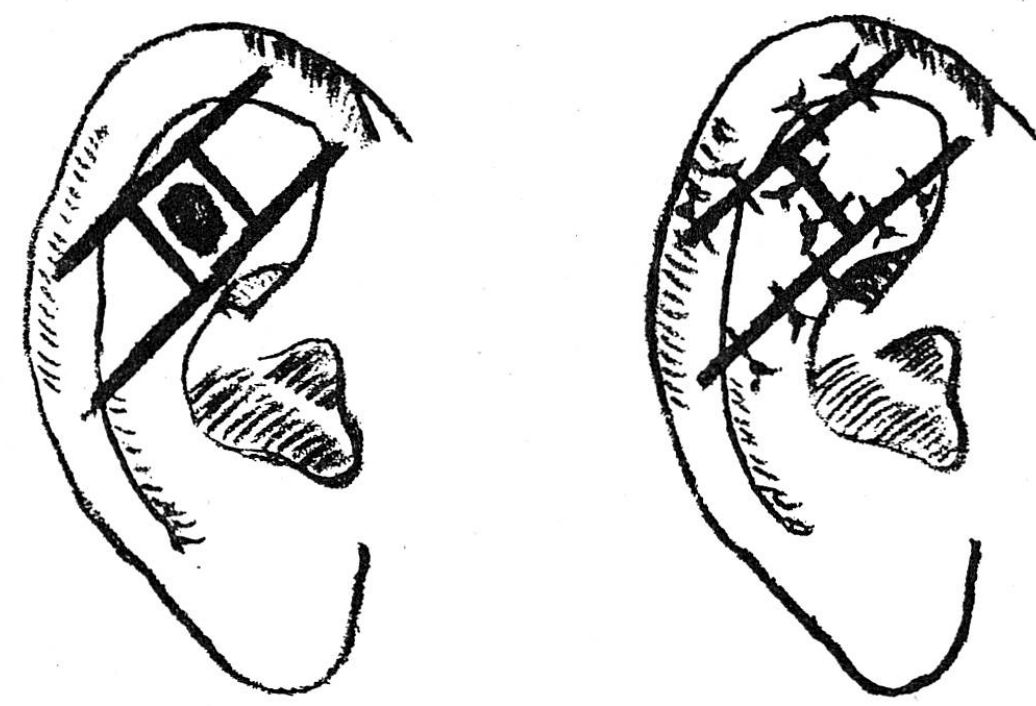

Abb. 10. Plastisches Verfahren nach Celsus und Antyll

schritt gegenüber der erwähnten Auflage von Mehlteig bedeutet der für Archigenes bezeugte Verband aus warmem mit Honig vermischten Brot, der, was seinen Zweck betrifft, Assoziationen zu den modernen plastischen Kunststoffverbänden hervorruft.

Das plastische Operationsverfahren des ANTYLL stimmt weitgehend mit der Celsischen Methode überein. Nach der Definition des Koloboms als Defekt, der entweder die ganze Dicke eines Teiles oder nur die bedeckende Haut betrifft ${ }^{59}$, gibt Antyll gemeinsam für die Kolobome an Augenbrauen, Stirn, Wange, Nase und Ohr folgende Anweisung (Abb.10): Der Defekt wird viereckig umschnitten und der obere und untere Rand nach beiden Seiten verlängert, «so daß alle Schnitte das Aussehen eines liegenden $I$ haben, wenn man sich vorstellt, daß dảs Viereck durch die Mitte des Buchstabens $\mathrm{H}$ dargestellt ist». Die Haut zwischen den Schnitten wird gelöst, nach der Mitte des Vierecks zusammengezogen und vernäht. Dabei sollen Weichteile mitgenommen werden, da die Haut sonst abstirbt. «Ist nicht nur die Haut», so fährt er fort, «sondern auch der Knorpel defekt, muß

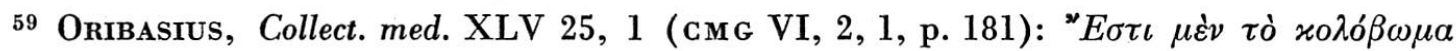

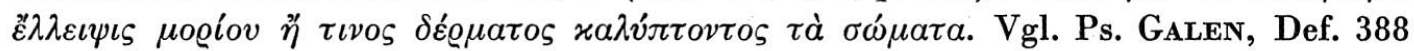
(XIX, 442, K.): Ko Vgl. M. Wellmann, Die pneumatische Schule, Philologische Untersuchungen 14, Berlin 1895, S. 80. 
vor der Vereinigung der Hautteile der Knorpel so weit excidiert werden, daß er sich möglichst gut der vorhandenen Öffnung des Koloboms anpaßt. Werden die Hautteile jetzt verbunden, wird das Ergebnis nicht häßlich sein. ${ }^{60}$

Der hohe Stand dieser in Alexandrien entwickelten plastischen Gesichtsoperationen setzt voraus, daß Traumen an Augenbrauen, Nase, Wangen, Lippen und Ohren häufig gewesen sein müssen. Die Erklärung, daß unter den erworbenen Defekten die Verletzungen der Boxer, Ringer, Allkämpfer und Gladiatoren einen erheblichen Anteil bildeten, bietet sich daher von selbst an.

Dies bestätigt Galen, der mehrfach mit Blick auf Faustkämpfer und Pankratiasten von Curta bzw. Kolobomata an Nase, Lippen und Ohren spricht ${ }^{61}$, der chirurgia curtorum allerdings nur einen gewissen kosmetischen Erfolg einräumen kann ${ }^{62}$. Zugerichtet wie die Liten Homers «lahm, runzlig und schielenden Blickes ${ }^{63}$ und in jedem Fall an irgendeinem Körperteil verstümmelt, führen die Athleten nach Galen ein Leben, das dem der Schweine ${ }^{64}$ gleicht. Er, der jahrelang Gladiatoren betreute, muß es aus eigenem Augenschein gewußt haben, auch wenn die Tendenz solcher Äußerungen im Protreptikos nicht zu verkennen ist.

Die Frage nach den Ohrverletzungen antiker Athleten hat damit eine eindeutige Antwort gefunden. Zeugnisse aus Literatur und Kunst bestätigen, daß Ohrmuscheltraumen häufig gewesen sind, wobei den Stiltendenzen und der jeweiligen künstlerischen Aussage entsprechend die Bildwerke keine wahrheitsgetreue Wiedergabe klinischer Erscheinungsbilder bieten.

Erst mit Beginn einer realistischen und exakt beobachtenden Kunst gestattet die künstlerische Darstellung eine genauere Diagnose über Art und Umfang der Ohrverletzungen. Immerhin darf es als legitim gelten, in «geschwollenen Ohren» antiker Bildwerke den Hinweis auf athletische Übung

60 Vgl. Celsus 7, 9, 4 (p. 326 MARx): Si cartilago in eo, quod incisum est, eminet, excidenda est: neque enim aut glutinatur aut acu tuto traicitur. Neque longe tamen excidi debet, ne inter duas oras liberae cutis utrimque coitus puris fieri potest.

${ }^{61}$ GaLeN, meth. med. 14, 16 (X, 1002, K.). Vgl. auch adhortatio c. 12 (I, 32, K.) und ad Thrasybulum c. 37 (V, 877, K.).

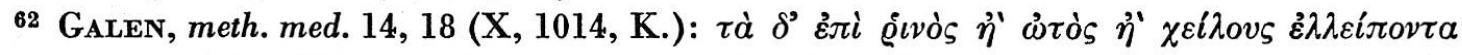

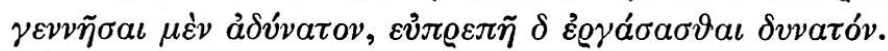

63 Ilias 9, 503. Vgl. GaLeN, de parv. pil. exercitatio c. 5 (V, 910, K.) und adhortatio c. 11 (I, 30, K.).

${ }^{64}$ Galen, adhortatio, c. 11 (I, 28 f., K.). 
zu erblicken ${ }^{65}$. Dies wird zusätzlich durch die literarisch erwähnten, archäologisch aber nicht belegbaren "Ohrschutzklappen» (å $\mu \varphi \omega \tau i \delta \delta \varepsilon)$ erhärtet, die allem Anschein nach aber nur im Training getragen worden sind ${ }^{66}$. Eine genaue Differenzierung freilich lassen auch literarische Zeugnisse nicht zu: Galen ${ }^{67}$ findet Entstellungen des Gesichts besonders bei Pankratiasten und Faustkämpfern. Philostrat ${ }^{68}$ führt « gebrochene Ohren » auf den Ringkampf zurück. Winckelmann spricht sogar vom «Pankratiastenohr». ${ }^{69}$ Die antiken Lexikographen wiederum sehen «Ohrbrüche» im Zusammenhang mit dem Faustkampf. Sicher dürfte sein, daß Ohrmuschelverletzungen in erster Linie bei Faustkämpfern eintreten. Daneben müssen natürlich auch Kriegs- und Berufsverletzungen in Betracht gezogen werden.

Fragt man nach den Beziehungen, die sich zwischen Kunst und medizinischer Literatur knüpfen lassen, so ergibt sich, daß mit Ausnahme der Karikatur keine Endzustände mit narbig geschrumpften Verkrüpplungsformen zur Darstellung kommen. Die operativen Verfahren innerhalb der chirurgia curtorum, wie sie in der Überlieferung durch Celsus und Antyll entgegentreten, finden begreiflicherweise in der Kunst keinen nachweisbaren Niederschlag. Sie scheinen überhaupt in Vergessenheit geraten zu sein und werden erst in der Neuzeit wieder aufgegriffen und" unter mannigfachen Modifikationen zur Anwendung gebracht. Ebenso begegnen in den Werken der Kunst keine Unterschiede zwischen behandelten und unbehandelten Othämatomen bzw. Perichondritiden. Dagegen lassen sich Beziehungen von Kunst und Medizin in der Beschreibung frischer Verletzungen aufzeigen. Prägnantestes Beispiel ist der Ohrknorpelbruch, den die hippo-

65 H.Sichtermann, Antike Plastik, Berlin 1965, Lieferung IV, Teil 9, S. 72, Anm. 7: «Freilich müßte untersucht werden, ob überhaupt geschwollene Ohren immer ein Hinweis auf die Palästra sein müssen.» Zur Frage vgl. auch J. Jürhner, Körperkultur im Altertum, Jenaer medizinhistorische Beiträge 12 (1928) 58: «Die durch den Faust- und Ringkampf verschwollenen Ohren sind ja immer ein Kennzeichen der Kämpfer gewesen und vielfach auch von der Kunst angedeutet worden. » Ferner J. ILBERg, Von der Heilkunde und Gesundheitslehre des Altertums, Münchner medizinische Wochenschrift 8 (1931) 322: «Häufig werden Verletzungen der Nase und der Ohrmuschel erwähnt, die Spuren davon vermögen wir ja noch jetzt an den Porträtstatuen der Athleten nachzuweisen.»

${ }^{66}$ Näheres bei J.H.Krause, a. a. O., S. 517.

${ }^{67}$ adhortatio c. 12 (I, 32, K.).

${ }^{68}$ Heroicus c. 12 b (p. 189, 27, KAYSER).

${ }^{69} \mathrm{Vgl}$. J. JüThNER, RE XVIII/3, 621, 51 ff. 
kratische Schrift De articulis erstmals erwähnt, dessen Existenz aber nachmals noch in der Pariser Schule Malgaigne bestreiten und Velpeau nur für den ossifizierten Altersknorpel einräumen sollte ${ }^{70}$.

70 J.F.Malgaigne, Abhandlungen der chirurgischen Anatomie und experimentalen Chirurgie, übersetzt von F. Reiss und J. LiehmanN, Prag 1842, I, S. 270: "Der Knorpel ist so dünn und geschmeidig, daß kein Beispiel seines Bruches existiert, obwohl alle Autoren des Altertums ihn beschrieben haben. Velpeau scheint ihn zuzugeben, wenn der Knorpel durch das Alter ossificiert ist. » 